\title{
Greenfield Gradual Migration Planning Toward Spectrally-Spatially Flexible Optical Networks
}

\author{
Piotr Lechowicz, Róża Goścień, Rubén Rumipamba-Zambrano, Jordi Perelló, \\ Salvatore Spadaro and Krzysztof Walkowiak
}

\begin{abstract}
This paper identifies the unavoidably required upgrade of short-term realizable elastic optical networks (EONs) operating over single-mode fibers toward spectrally-spatially flexible optical networks (SSFONs), exploiting spatial division multiplexing (SDM) technology in order to cope with the expected midand long-term future traffic forecasts. Since a complete EON to SS-FON network migration becomes unrealistic overnight, this paper proposes a gradual greenfield migration strategy employing novel heuristic planning solutions. These solutions allow identifying which network components should be SDM-capable ones, so as to support the forecasted traffic increase at given time. To allow transmission in SS-FON, links should be extended with SDM-capable fibers and nodes should be equipped with SDM-capable re-configurable optical add and drop multiplexers (ROADMs). Using the proposed solutions, a migration case study in a national reference core network is provided and analyzed.
\end{abstract}

Index Terms-spectrally-spatially flexible optical networks; migration planning; elastic optical networks

\section{INTRODUCTION}

Network traffic has been growing at huge speed over last few decades and across all network regions, from the optical core to fixed and mobile access. The major reason behind this increase is the huge popularity of services, applications and technologies including cloud computing, contentoriented networking, network virtualization and softwarization. All these trends trigger the need for developing new optical network architectures and technologies. Recently, the scientific research community society has identified a flexible (elastic) optical network (EON) architecture for future optical transport networks due to its numerous advantages. EONs divide available spectral resources into narrow frequency slots tightly assigned to connections according

P. Lechowicz, R. Goścień and K. Walkowiak are with Department of Systems and Computer Networks, Wrocław University of Science and Technology, Wrocław, Poland.

R. Rumipamba-Zambrano, J. Perelló and S. Spadaro are with Universitat Politècnica de Catalunya (UPC), Barcelona, Spain. to their bandwidth requirements. Flexible assignment of spectral resources allow for higher elasticity in the connection assignment compared to fixed-grid (wavelength division multiplexing, WDM) networks. Moreover, EONs allow for high-bandwidth multi-carrier transmission consisting of multiple optical carriers and adaptive use of various modulation formats according to the optical path properties [1] $-[3]$. However, despite the increased flexibility in resource management, available capacity in single-core singlemode fibers (SMFs) used in EONs can approach their upper bound, as indicated by the nonlinear Shannon limit [4]. To overcome such a future capacity crunch, researchers propose new promising optical architecture improvement — implementation of space division multiplexing (SDM). In a nutshell, SDM introduces a new spatial dimension to enable parallel optical signal transmission through spatial modes co-propagating in suitably designed optical fibers. Although EON and SDM are orthogonal technologies in principle, their simultaneous application provides a promising solution called spectrally-spatially flexible optical network (SS-FON), which combines benefits of both approaches - operations within flexible frequency grids and spatial multiplexing. In this paper, we propose a greenfield migration plan from a conventional EON towards an SS-FON, where in every assumed period of time (e.g., every 3 years) minimization of the number of inserted SDM-capable fibers and devices is sought, enabling to support the forecasted traffic volume.

\section{Migration to SS-FON ARchitecture}

As a short term solution, a migration from fixedto flexible- (elastic) grid is sufficient [2]. Nevertheless, the capacity crunch in EONs is unavoidable, hence the migration toward SS-FON is an inevitable consequence. In order to enable spatial division multiplexing in EONs, first of 


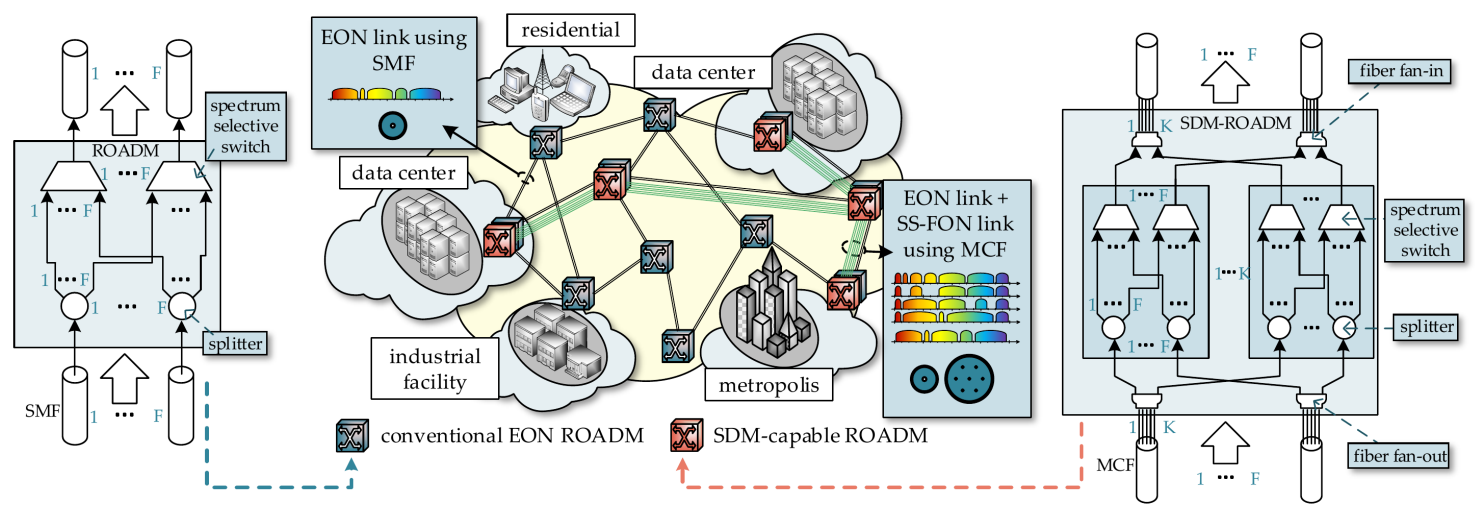

Fig. 1. Overview of a partially migrated network toward SS-FON.

all, the communication media supporting parallel transmission is required; moreover, network nodes should be equipped with devices capable of switching spatially multiplexed traffic. As the whole network upgrade will demand significant expenditures, operators will tend to extend the migration process over time. Thus, it is crucial that the traffic can be allocated in partially migrated network scenarios.

\section{A. Fibers Supporting SDM}

Several types of fibers are proposed supporting multiplexing in the spatial domain, e.g., singlemode fiber bundle (SMFB) - multiple SMFs aggregated in the bundle, or multi-core fibers (MCFs) - multiple cores embedded in a single fiber cladding. For the sake of simplicity, we refer to the utilized spatial resources as spatial modes, regardless of whether the term "mode" refers to multiple SMFs or cores in MCFs [5]. MCFs are prone to inter-core crosstalk (IC-XT) which is the amount of signal leaking from adjacent cores to currently considered one, when transmitting signals in overlapping spectrum regions. After exceeding an acceptable IC-XT level, the proper reception of signal is impossible. Therefore, IC-XT may significantly affect the transmission reach, and in consequence, the network performance [6]. In fact, depending on the mean crosstalk increase per length unit of fiber, various modulation formats may be applied on shorter transmission reaches, and in consequence, higher number of migrated components may be required to compensate the usage of less spectrally efficient modulation formats to serve the same requests [7]. Therefore, it is crucial during network planning to select MCFs with appropriate characteristic of IC$\mathrm{XT}$ propagation to realize transmission. Despite
MCFs' drawbacks related to IC-XT, MCFs are a promising solution for future SS-FONs, as they are characterized by lower diameter and higher spatial effieciency when compared to SMFBs, i.e., provided capacity per $\mathrm{mm}^{2}$.

\section{B. Nodes Architecture}

Possible ROADM designs for EONs are based on Broadcast \& Select $(B \& S)$ or Route \& Select (R\&S) architectural concepts [8]. As in Fig. 11. $\mathrm{B} \& \mathrm{~S}$ places a $1 \times F$ power splitter per input fiber, which broadcasts incoming optical signals to all $F$ output fibers, where $F \times 1$ Spectrum Selective Switch (SSS) devices are also placed allowing to switch any spectral portion from any input to any output port. In this way, SSSs can select which incoming signals are further transmitted over each output fiber. Compared to $B \& S, R \& S$ replaces the $1 \times F$ power splitters by $1 \times F$ SSSs. Input signals are not broadcasted to all output ports anymore, but directly switched to the desired output fiber.

Both B\&S and R\&S architectural concepts can be extended to SS-FONs. However, to achieve full interconnection between input and output ports an enormous complexity can appear, for example, resulting from the increased number of needed SSS devices and port count. Therefore, ROADMs may introduce additional fiber/core continuity constraints to reduce the complexity, i.e., lacking of Lane Change (LC) support. In such case, when properly indexing spatial modes of the fiber, lack of LC assumes that each spatial mode of input fiber may be only switched to the spatial mode of the output fiber with the same index. Fig. 1 shows B\&S implementation for SDM-ROADM realizing Independent-Switching (Ind-Sw) architecture without LC support. Ind$\mathrm{Sw}$ allows to accommodate and switch requests 
per spatial modes basis, i.e., request may be routed using one of any available spatial modes preserving core continuity constraint. As shown in Fig. 1, $K$ times $(F \times 1)$ SSSs are required per output SDM fiber. Alternative SDM-capable ROADM architectures have also been proposed in the literature, trading flexibility (i.e., network performance), for lower complexity/costs (e.g., see [9]). In particular, the flexibility may be decreased realizing Joint-Switching (J-Sw) or Fractional Joint-Switching ( $\mathrm{FrJ}-\mathrm{Sw}$ ) architectures. In J-Sw, all spatial modes are switched together as a single entitiy. In FrJ-Sw, spatial modes are divided into groups, wherein spatial modes inside a given group have to be routed together, however, particular groups may be switched independently. It is worth noting, that FrJ-Sw may be seen as a trade-off with higher switching flexiblity than JSw and lower device complexity than Ind-Sw. In each switching architecture, i.e., Ind-Sw, FrJ-Sw and J-Sw, the core continuity constraint may be applied achieving node complexity reduction in exchange for a performance penalty (e.g., 15\% at most for InS without LC support against a fully flexible ROADM [9]).

\section{Greenfield Migration}

Despite significant one-time cost, the whole network upgrade is time-consuming and it may not be possible to apply it without network operating interruption. What is even more important, it may be prone to unforeseen errors, leading to transient network performance degradation or a total network inoperability. Thus, an important question arises, whether it is possible to operate with a partially upgraded network. In this paper, we assume that each network link is equipped with a SMF and each network node with a conventional ROADM. Parallel transmission in a given link is possible only when a new MCF is deployed and both end nodes are equipped with additional SDM-capable ROADMs. In particular, we assume that both unidirectional links between certain node pairs are equipped with MCFs at the same time. In such case, we assume that EON with SS-FON network are co-existing and traffic is realized in one of the available networks. Fig. 1 presents an overview of partially migrated network. Major locations, i.e., data centers (DCs) and metropolis are connected together using a high-capacity SS-FON, allowing for huge bandwidth transmission. As the next step, the industrial facility may also request an upgrade of their nearest infrastructure in order to achieve higher bandwidth access to DCs.

\section{OptimizATION ISSUES}

One of the fundamental issues that appears in SSFONs is the routing, space and spectrum allocation (RSSA) problem, which refers to finding for each pending request a routing path and suitable optical corridor conducted on selected available spatial resources accounting for the continuity, contiguity and spectrum non-overlapping constraints in the spectrum domain [10]. Moreover, we assume the continuity constraint in the spatial domain. Finding a suitable optical corridor may be especially problematic in SMFs, resulting from existing bottlenecks decreasing the network performance in terms of provisioned traffic. At some point, a crucial decision emerges - which network components should be selected first in order to provision forecast traffic and minimize the cost, i.e., delay the cost of whole network upgrade.

According to assumptions in Section II-A, the network is equipped with SMFs and the key components to be deployed are MCFs. However, in order to enable SDM transmission through MCFs, ROADMs at both ends of a fiber link have to be SDM-capable. Thus, during the migration execution, it is required not only to consider fibers, but also to track which nodes should be equipped with SDM-capable ROADMs. We assume that the cost of fiber deployment is significantly higher than the cost of an SDMcapable ROADM. Therefore, an optimal selection of fibers to be upgraded from SMF to MCF is targeted, directly equipping SDM-capable ROADMs at their remote endpoints as needed. Beside, it is also required to allocate the traffic in the network. For that purpose, we apply well-known Most Subcarriers First (MSF) heuristic [10].

\section{A. Optimization Algorithm}

To solve the migration planning problem, we propose an Stochastic Iterated Local Search (SILS) algorithm, with the aim to minimize the number of required upgraded components while provisioning the whole forecast traffic in the network. Components for upgrade are selected incremen- 
tally, i.e., every 3-year interval certain components are selected for upgrade and cannot be downgraded in the future years, as it is assumed to be not beneficial.

The initial solution for SILS is constructed using another heuristic successfully studied in our previous work related to brownfield migration, i.e., Link with Highest Subcarriers First (LCS) [11]. It consecutively replaces fibers and nodes in the network until the whole traffic can be accommodated applying the following strategy. Firstly, the simulation is run to allocate the traffic in the network using the MSF heuristic. At this step, it is assumed that fibers have unlimited capacity, i.e., there is no restriction in the number of available frequency slots in each fiber. This assumption allows to investigate how the traffic is distributed in the network (if limited bandwidth had been assumed, part of the traffic would have been blocked due to the insufficient spectral resources). Next, the algorithm checks whether allocated traffic exceeds actual maximum capacity of the fiber, e.g., $4 \mathrm{THz}$. In such case, the need to deploy new MCFs emerges to increase the network capacity. The algorithm checks what is the summary traffic evaluated in subcarriers allocated on each SMF link. Next, the link with the highest value of that metric is equipped with $\mathrm{MCF}$ and nodes connected to that link are equipped with SDMcapable ROADMs. Note, one of these nodes may be already upgraded, due to the selection of some other link connected to that node, thus it is required for algorithm to track these changes.

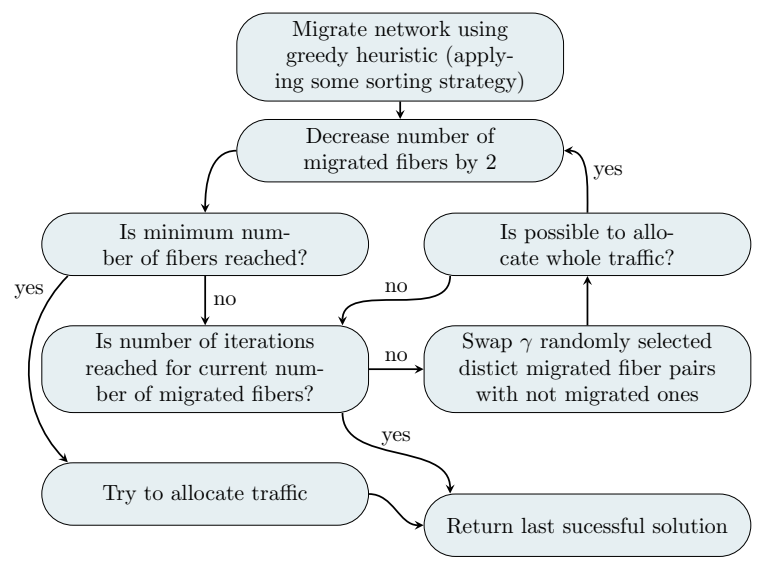

Fig. 2. Flowchart of SILS metaheuristic.

The above-mentioned LCS heuristic may be used to provide an initial solution; however, deterministic algorithms may often get stuck in local optima. Contrarily, metaheuristics are capable of escaping from such points through the introduction of randomness to their execution. Therefore, we use the SILS metaheuristic which iteratively tries to find a feasible solution for given number of migrated fibers by random replacement of $\gamma$ elements of the solution set, i.e., pair of fibers (see Fig. 2). Here, $\gamma$ is the integer parameter determining how many pairs of fibers are replaced at each iteration. As the first step, the initial solution is obtained with the above-mentioned LCS heuristic. Next, the number of migrated links is consecutively decreased by 2 , and for each value of migrated links multiple solutions are iteratively created until it is possible to allocate the whole traffic in the network, or the maximum number of iterations is reached. In the former case, at each iteration a new solution is created replacing $\gamma$ randomly selected migrated fiber pairs (i.e., two unidirectional fibers at given link) with $\gamma$ randomly selected not-migrated ones. When it is possible to accommodate the whole traffic, the solution is stored as a feasible one and the algorithm further decreases the number of migrated fibers hoping to find another feasible solution of better quality (i.e., for lower number of migrated fibers). For new considered number of migrated fibers, the above solutions creation steps are repeated. In the latter case, when the maximum number of iterations for a given number of migrated fibers is reached, the algorithm stops execution and returns the last feasible result. The stopping criterion is defined by the maximum number of non-improving iterations.

\section{Case Study}

In order to illustrate the migration planning toward SS-FONs, we show below a case study. The simulations are run on a representative network topology - DT14 German national network containing 14 nodes and 46 links, with an average link length of $182 \mathrm{~km}$ (see Fig. 5; highlighted nodes represent DCs). The SILS migration planning metaheuristic described in the previous section is applied to run the simulations

\section{A. Key Assumptions}

In the considered network, nodes are connected with unidirectional links. Initially, each network node is equipped with a conventional ROADM and each link contains two unidirectional SMFs. SS-FON transmission is enabled only when a 
link deploys two unidirectional MCFs, and at the same time, nodes at both ends of the link are equipped with SDM-capable ROADMs. In such case, we assume that the link comprises both fibers and two network topologies are co-existing in parallel. During the experiments, we consider $12-\mathrm{MCF}$ with worst aggregated IC-XT equal to $61.9 \mathrm{~dB} / \mathrm{km}$. Each SMF, and MCF core, provides $4 \mathrm{THz}$ of available spectrum divided into small frequency slots of $12.5 \mathrm{GHz}$ width (equivalent to 320 slots). Each node is equipped with distanceadaptive coherent transceivers that support reconfigurable bit-rates and various modulation formats (MFs) according to the optical path length. In particular, the supported transmission reach is equal to $6300,3500,1200$ and $600 \mathrm{~km}$ for BPSK, QPSK, 8-QAM and 16-QAM, respectively [12]. Each transceiver operates at a fixed baud rate of 28 GBaud and transmits/receives an optical carrier (OC) occupying a spectral width of 3 frequency slots $(37.5 \mathrm{GHz})$. The bit-rate supported by single transceiver depends on the applied MF and is equal to 50, 100, 150 and 200 Gbps for BPSK, QPSK, 8-QAM and 16-QAM, respectively [13]. If the requested bit-rate exceeds a single transceiver capacity, signal may be transmitted using several adjacent transceivers creating a spectral super-channel (SCh) or spectral-spatial SCh, depending on the applied switching policy. Each SCh has to be separated from adjacent ones by means of a guardband of 1 frequency slot width. In the SS-FON network, we consider scenarios employing different switching architectures, i.e., Ind-Sw, J-Sw and FrJ-Sw. Moreover, for FrJ-Sw we consider cases with groups of cores equal to $G=\{6,4,3,2\}$. We assume that regardless of the selected architecture, LC operations are not supported.

\section{B. Traffic Forecast}

Service demands model is applied for a period of 12 years from 2019 to 2031, based on the information provided in the Cisco's report [14]: (i) city-to-city - a unicast request between two end nodes in the network; (ii) city-to-dc - an anycast request between any of the available DCs and the client node; or in the opposite direction, from a client node to any of the available DCs; (iii) dc-to-dc - a unicast request between two
DCs in the network (the communication between DCs is realized in the backbone network).

TABLE I

EVOLUTION OF TRAFFIC OVER TIME

\begin{tabular}{|c||cccr|}
\hline \multicolumn{1}{|c||}{} & \multicolumn{4}{c|}{$\begin{array}{c}\text { DT14 network } \\
\text { Traffic volume (Tb/s) }\end{array}$} \\
\cline { 2 - 5 } Year & City to city & City to DC & DC to DC & Total \\
\hline 2019 & 3.4 & 21.8 & 14.8 & 40.0 \\
2022 & 4.8 & 42.7 & 34.7 & 82.1 \\
2025 & 6.7 & 83.7 & 81.0 & 171.5 \\
2028 & 9.4 & 164.2 & 189.4 & 363.0 \\
2031 & 13.2 & 321.8 & 442.9 & 777.9 \\
\hline CAGR [\%] & 12.1 & 25.2 & 32.7 & 28.1 \\
\hline
\end{tabular}

In more detail, the initial ratio of abovementioned request types and the compound annual growth rate (CAGR) are evaluated based on the CISCO prediction (see Tab. II). The initial traffic is equal to 50 Tbps which allows to saturate the network in later years. It is worth noting that in different years, different traffic distribution is provided to the network as different traffic types increase with different CAGR. In particular, in latter years, dc-to-dc traffic becomes a dominant one [15].

\section{Results}

The main performance metric used in the simulations is the required number of migrated fibers. The tuning of the SILS metaheuristic has been initially performed and the best found parameters has been selected. The simulations are run for various switching paradigms and algorithms and various number of DCs $R$. In all experiments, the number of candidate paths between node pairs is set to 10. All experiments are averaged over 30 traffic matrices, and the confidence intervals are presented within $95 \%$ confidence intervals.

Fig. 3 reports comparison of various switching paradigms, namely, Ind-Sw, J-Sw and FrJ-Sw. According to results, for earlier years, there is no significant performance differences among them. In 2031, it can be observed significant performance decrease for J-Sw. The reason comes from the fact that small requests are not effectively utilizing available resources as all spatial modes are switched as a single entity. Nevertheless, it is sufficient to apply FrJ-Sw with 3 or 4 groups to achive significant performance increase in terms of traffic allocation, and indirectly, in network migration performance. In particular, FrJ-Sw with 3 groups decreases migration ratio by $47.8 \%$ when compared to J-Sw in 2031. 


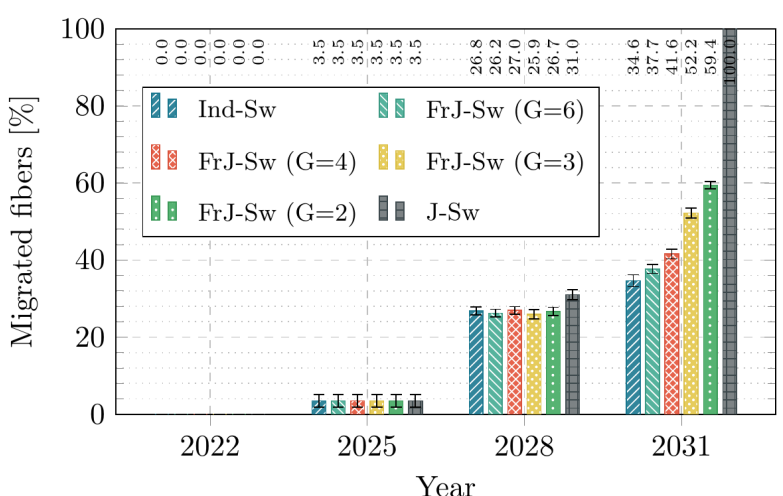

Fig. 3. Percentage of migrated fibers along years for various switching paradigms for SILS algorithm.
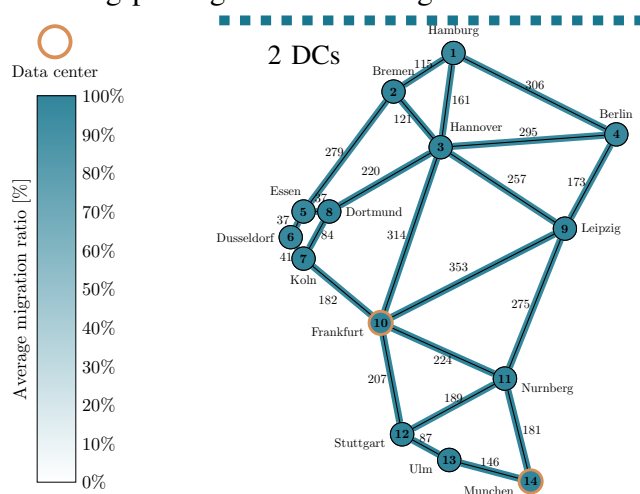

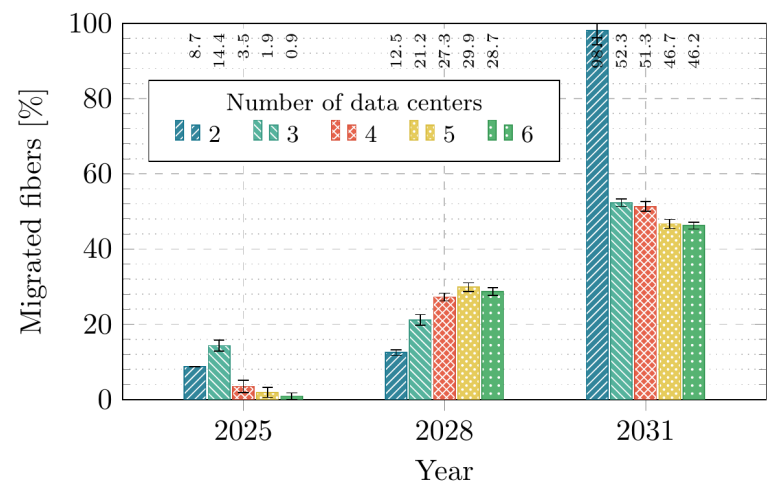

Fig. 4. Percentage of migrated fibers along years for various number of data centers for SILS algorithm.

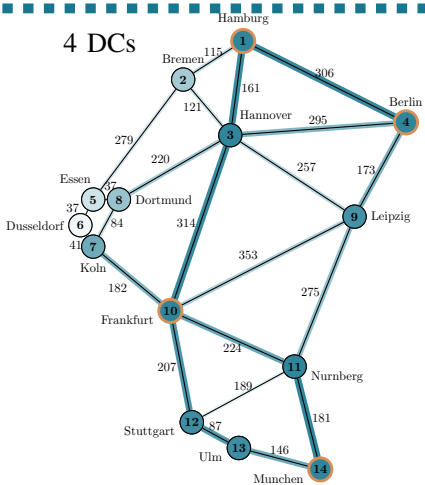

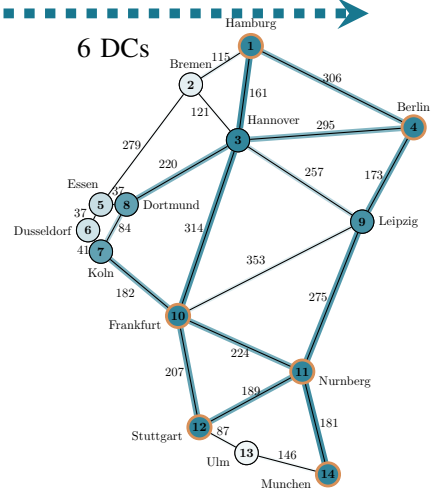

Fig. 5. Average fibers and nodes migration ratio for 2028 for various number of DCs.

Next, the migration performance is investigated for various numbers of DCs, i.e., $|R|=$ $\{2,3, \ldots, 6\}$. Fig. 4 reports migrated fibres percentage for various DC number along the years assuming 3 groups of spatial modes in MCFs. Firstly, for each number of DCs, migration ratio is increasing in time. It is intuitive, as the summary traffic is also increasing one year after another, and higher amount of spatial resources may be needed. However, the migration has different performance for different number of DCs and it is varying in different years. In particular, in mid years, higher number of DCs yields worse performance, while in latter years the trend is opposite. It is worth noting that different number of DCs corresponds to different traffic distribution. Moreover, the CAGR of DC-to-DC traffic $(32.7 \%)$ is the highest, making this type of traffic the major one in later years. Taking this into account, communication between DCs and between DCs and client nodes may significantly change network performance. In particular, with higher number of DCs, it may be required to migrate a higher number of links (and in consequence nodes) to connect all DCs in the network and establish the required traffic between them. Con- versely, when number of DCs is small, communication between client nodes and DCs may require longer paths, which results in selecting worst MFs and a higher number of required subcarriers to establish the demand. In Fig. 5, average migration ratio of all links and nodes for various number of DCs is presented for 2031. In more detail, results are averaged over 30 traffic matrices, and presented values correspond to the frequency of given link/nodes selection for migration. For example $50 \%$ denotes that given link/node is migrated in 15 from all 30 simulations. As it can be observed, when 2 DCs are assumed, whole network requires migration in order to reach DCs by distant clients. For 4 DCs, lower number of nodes is migrated, allowing for high-bandwidth communication between DCs. Finally, for 6 DCs, this number decreases even further as city-to-DC connections are realized on shorter distance using more spectrally efficient MFs.

\section{CONCLUSION}

This paper has addressed the required upgrade of EONs over SMF that should be deployed in shortterm optical core network scenarios toward SSFONs leveraging SDM, as a mean to overcoming the future SMF capacity crunch. Novel heuristic 
planning solutions for greenfield gradual EON to SS-FON migration are proposed and evaluated in DT14 national network topology, assuming various switching paradigms. The obtained results depict that applying more complex and expensive switching architectures influence the percentage of deployed fibers, and ROADMs indirectly, per time period in later years. Additionally, a strong influence is observed between the number of DCs available in the netowrk and the migration performance.

\section{ACKNOWLEDGMENT}

The work of P. Lechowicz, R. Goścień and K. Walkowiak was supported by National Science Centre, Poland under Grant 2015/19/B/ST7/02490. The work of R. RumipambaZambrano was supported by SYNESCYT - Ecuador. The work of J. Perelló and S. Spadaro was supported by the ALLIANCE project (TEC2017-90034-C2-1-R/C2-2-R).

\section{REFERENCES}

[1] M. Jinno et al., "Spectrum-efficient and scalable elastic optical path network: architecture, benefits, and enabling technologies," IEEE Com. Mag., vol. 47, no. 11, 2009.

[2] M. Ruiz et al., "Planning fixed to flexgrid gradual migration: drivers and open issues," IEEE Com. Mag., vol. 52, no. 1, pp. 70-76, 2014.

[3] P. Lu et al., "Highly efficient data migration and backup for big data applications in elastic optical inter-data-center networks," IEEE Network, vol. 29, no. 5, pp. 36-42, 2015.

[4] P. J. Winzer, "Spatial multiplexing in fiber optics: The 10x scaling of metro/core capacities," Bell Labs Techn. J., vol. 19, pp. 22-30, 2014.

[5] G. M. Saridis et al., "Survey and evaluation of space division multiplexing: From technologies to optical networks," IEEE Commun. Surv. Tutor, vol. 17, no. 4, pp. 2136-2156, 2015.

[6] H. Yang et al., "Resource assignment based on dynamic fuzzy clustering in elastic optical networks with multi-core fibers," IEEE Transac. Com., vol. 67, no. 5, pp. 3457-3469, 2019.

[7] P. Lechowicz et al., "Inter-core crosstalk impact on migration planning from elastic optical networks to spectrallyspatially flexible optical networks," in OFC 2019, 2019.

[8] B. Collings, "New devices enabling software-defined optical networks," IEEE Com. Mag., vol. 51, no. 3, pp. 66-71, 2013.

[9] R. Rumipamba-Zambrano et al., "Space continuity constraint in dynamic flex-grid/sdm optical core networks: An evaluation with spatial and spectral super-channels," Com. Com., vol. 126, pp. 38-49, 2018.

[10] M. Klinkowski et al., "Survey of resource allocation schemes and algorithms in spectrally-spatially flexible optical networking," Opt. Switch. Netw., vol. 27, pp. 58-78, 2018.

[11] P. Lechowicz et al., "Migration Planning from Elastic Optical Networks to Spectrally-Spatially Flexible Optical Networks," in PSC 2018, 2018.

[12] P. S. Khodashenas et al., "Comparison of spectral and spatial super-channel allocation schemes for sdm networks," J. Lightwave Technol., vol. 34, no. 11, pp. 2710-2716, 2016.
[13] C. Rottondi et al., "Optical ring metro networks with flexible grid and distance-adaptive optical coherent transceivers," Bell Labs Techn. J., vol. 18, no. 3, pp. 95-110, 2013.

[14] CISCO, "Cisco Visual Networking Index: Forecast and Methodology, 2016 - 2022,” Tech. Rep., 2017.

[15] M. Klinkowski and K. Walkowiak, "On the advantages of elastic optical networks for provisioning of cloud computing traffic," IEEE Network, vol. 27, no. 6, pp. 44-51, 2013.

\section{BIOGRAPHIES}

PIOTR LECHOWICZ received his M.S. degree in computer science from the Wroclaw University of Science and Technology (WUST), in 2016. He is currently working toward his Ph.D. degree in computer science in the Department of Systems and Computer Networks, WUST. His research interests include modeling and optimization of spectrally-spatially flexible optical networks.

RóżA GoŚCIEŃ received the Ph.D. degree in computer science from Wroclaw University of Science and Technology (WUST), Wroclaw, Poland, in 2016. She is currently an Assistant Professor with the Department of Systems and Computer Networks, WUST. Her current research focuses on the modeling and optimization of survivable communication networks.

RubÉn RUMipamba-ZAMBRANo received the M.Sc. (2014) and Ph.D. (2019) degrees from Universitat Politècnica de Catalunya (UPC) - Spain. He has worked for about 6 years at the Corporación Nacional de Telecomunicaciones (CNT E.P.) - Ecuador, as senior Telecom engineer. His research interests include IP and future optical networks with emphasis in planning, design and operations.

JoRdi PERElló received the M.Sc. (2005) and Ph.D. (2009) degrees from the Universitat Politècnica de Catalunya (UPC). He holds a tenured Assistant Professor position at the UPC. His current research interests target the performance optimization of $5 \mathrm{G}$ data transport networks. He has co-authored around 100 papers, more than 40 of them published in JCR-ranked journals and magazines.

SALVATORE SPADARO received the M.Sc. (2000) and the Ph.D. (2005) Degrees in Telecommunications Engineering from Universitat Politècnica de Catalunya (UPC). His research interests are in the fields of all-optical networks, optical technologies for $5 \mathrm{G}$, planning and operation of multi-core/SDM optical networks. Dr. Spadaro has published more than 180 articles in international journals and conference proceedings.

KRZYSZTOF WALKOWIAK received the Ph.D. and the D.Sc. (Habilitation) degrees from Wroclaw University of Science and Technology (WUST), Wroclaw, Poland, in 2000 and 2008, respectively. He is currently a Professor at the Department of Systems and Computer Networks, WUST. His research interest focuses on optimization of communication networks, survivability, cognitive optical networks. He has co-authored above 240 papers, 50 of them published in JCR journals. 